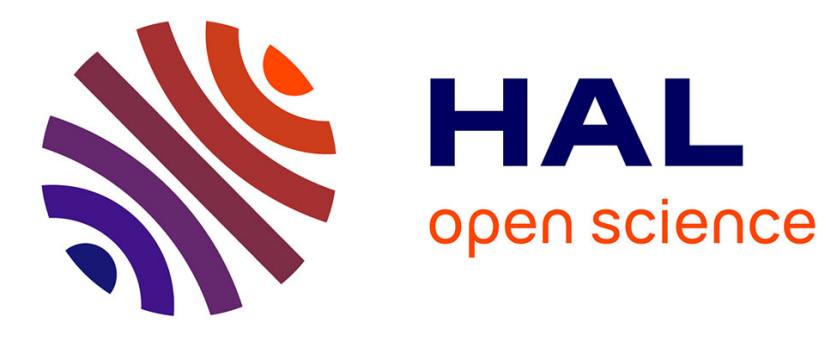

\title{
Pour introduire à la lecture de Quine
} Joseph Vidal-Rosset

\section{To cite this version:}

Joseph Vidal-Rosset. Pour introduire à la lecture de Quine. Jean-Maurice Monnoyer. Lire Quine Logique et ontologie, Éditions de l'éclat, 2006, 2841621138. 10.3917/ecla.monno.2006.01.0029 hal01242368

\section{HAL Id: hal-01242368 https://hal.science/hal-01242368}

Submitted on 11 Dec 2015

HAL is a multi-disciplinary open access archive for the deposit and dissemination of scientific research documents, whether they are published or not. The documents may come from teaching and research institutions in France or abroad, or from public or private research centers.
L'archive ouverte pluridisciplinaire HAL, est destinée au dépôt et à la diffusion de documents scientifiques de niveau recherche, publiés ou non, émanant des établissements d'enseignement et de recherche français ou étrangers, des laboratoires publics ou privés. 


\section{Pour introduire à la lecture de Quine Joseph Vidal-Rosset}

Comme le souligne Gloria Orrigi' ${ }^{1}$, Quine est un penseur systématique qui n'a pas limité son activité philosophique à la seule analyse ponctuelle de problèmes singuliers - bien qu'il ait évidemment encouragé ce type de travail dans la tradition analytique -, mais il a construit un véritable système de thèses philosophiques liées les unes aux autres et pour lequel, à l'instar du système de Leibniz, il n'existe pas vraiment une voie d'accès principale ou plus fondamentale que les autres. C'est un bâtiment où les quartiers communiquent entre eux. On jugera ici de la solidité de l'édifice par la simple exposition de sa structure. On soulignera les thèses les plus fortes qui sont comme l'armature du navire (selon une comparaison chère à Quine). Je propose donc ici à la fois une invitation à découvrir les questions soulevées par Quine et un exposé des résultats de sa philosophie².

Si l'on devait cependant privilégier un thème dans le système du philosophe de Harvard, celui de la vérité et de la signification s'imposerait. Quine fut, après Frege et Russell, à l'origine des réflexions sur cette question dominant les préoccupations des philosophes analytiques contemporains. La redéfinition de l'empirisme et le naturalisme sont les thèses principales de son épistémologie. Le behaviorisme offre la méthode d'une analyse de la signification à l'intérieur duquel la remise en cause de la distinction kantienne de l'analytique et du synthétique et la thèse de l'indétermination de la traduction prennent sens. L'analyse logique de l'ontologie et 
de sa relativité ayant toujours été faite par Quine dans un esprit naturaliste ${ }^{3}$, j'adopterai le plan suivant:

1. L'empirisme et la naturalisation de l'épistémologie

2. La reconstruction behavioriste de la signification

3. Logique et ontologie

\section{L'empirisme et la naturalisation de l'épistémologiet}

L'empirisme est une thèse épistémologique qui affirme que le mode d'accès aux connaissances, quel que soit le degré d'abstraction de celles-ci, a toujours une origine sensorielle. La naturalisation de l'épistémologie est principalement une thèse anti-cartésienne. Quine est de ceux, «- large minorité ou petite majorité - qui répudient le rêve cartésien d'une fondation pour la certitude scientifique plus solide que la méthode scientifique elle-même ${ }^{5}$ ». Pour l'épistémologie naturelle, il n'existe pas de philosophie première. Comme le montrent les premières phrases de La poursuite de la vérité, l'empirisme offre à l'épistémologie ce problème à résoudre: «Partant des impacts sur nos surfaces sensorielles, nous avons fait jaillir par notre création collective et cumulative, au fil des générations, notre théorie systématique du monde extérieur. Notre système a prouvé sa réussite en annonçant de nouvelles entrées sensorielles. Comment avons-nous procédé6?» L'attitude entièrement conséquente avec la thèse de la naturalisation de l'épistémologie serait, pour répondre à cette question, de laisser désormais la place aux sciences positives que sont la neurologie, la psychologie expérimentale, la psycholinguistique, la génétique évolutionniste et, accessoirement, à l'histoire des sciences.

Des philosophes verront dans cette absence d'une philosophie première le risque de l'absence de la philosophie tout court; l'épistémologie naturelle serait en fait une épistémologie non philosophique. On répondra à cette inquiétude en soulignant que le système de Quine est une philosophie de la connaissance qui offre un schéma de compréhension de la façon dont les théories - à l'épreuve de la prédiction et sur la base 
de l'expérience empirique -, nous permettent de comprendre le monde extérieur. Dans ce schéma, la chaîne causale de la connaissance est décrite en termes d'entrée-sortie (input-output): les excitations des récepteurs nerveux au début de la chaîne, les théories scientifiques à l'autre bout; entre les deux la complexité des phénomènes neuronaux et, parmi eux, ceux qui correspondent aux situations spécifiquement linguistiques ${ }^{7}$. La philosophie n'étant pas fondamentalement différente de la science, l'épistémologie naturelle est scientifique et l'empirisme auquel elle est associée l'est également ${ }^{8}$, car c'est sur l'évidence sensorielle ( sensory evidence) que se fondent finalement les sciences de la nature pour dire ce qui existe.

Par «évidence sensorielle», Quine entend la stimulation des récepteurs sensoriels ${ }^{9}$, rien de plus. Gibson rappelle en effet que Quine a récemment écrit que, dans sa «théorie de l'évidence, le terme d'évidence ne donne aucune explication et ne joue aucun rôle ${ }^{10}$ ». Or cette absence de développement sur le rôle de l'évidence est philosophiquement limpide: en parlant des récepteurs nerveux et leurs stimulations plutôt que des sens et des données sensibles, Quine reconnaît ouvertement vouloir s'éloigner de toute référence au sujet pensant, d'où l'inutilité du terme d'évidence dès lors qu'on l'entend dans le contexte de la tradition cartésienne ${ }^{11}$.

Tout comme Russell a distingué la connaissance par expérience directe (knowledge by acquaintance) de la connaissance par description, Quine s'est attaché à décrire le premier stade non verbal de la connaissance du monde extérieur et son lien avec des énoncés occasionnels et singuliers ${ }^{12}$. En 1960, dans Word and Object, il utilise l'expression de significationstimulus (affirmative ou négative) pour désigner la classe de toutes les stimulations qui dicteraient l'acquiescement ou le rejet d'un énoncé d'observation quelconque pour un locuteur donné13. Un énoncé d'observation peut être défini comme une émission verbale occasionnelle, telle «il pleut», sur laquelle les locuteurs d'une même langue peuvent s'entendre au vu d'une situation (stimulation) partagée. Les 
énoncés d'observations sont à la fois les premiers outils de l'apprentissage du langage, qui s'inculque et se contrôle socialement, et le véhicule de l'apport empirique d'une science: sans ces éléments premiers, il n'y aurait nul moyen de s'élever aux généralisations composées de données observables que sont les énoncés catégoriques d'observations (ces théories scientifiques miniatures du type «chaque fois où

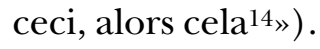

L'expression de «signification-stimulus » (stimulus-meaning) est remplacée dans Pursuit of Truth (1990) par celle de parcours stimulatoire (stimulus range), suite à une remarque de Føllesdal, selon laquelle les significations relèvent du domaine public. Ce changement terminologique est une précision apportée à la théorie initiale de Word and Object comme le montre le texte suivant:

Des énoncés d'observation sont stimulatoirement équivalents pour un locuteur si leur parcours stimulatoires sont pour lui les mêmes. Mais alors que les stimulations de chacun et leur parcours sont choses privées, l'équivalence stimulatoire fait sens socialement. Des énoncés sont stimulatoirement équivalents pour la communauté s'ils le sont pour chacun de ses membres. Cela n'est néanmoins pas valable de langue à langue, à moins que la communauté ne soit bilingue ${ }^{15}$.

On retrouve ainsi la possibilité de l'équivalence entre «Gavagai » et «Lapin» si les deux expressions ont, dans le vocabulaire de Word and Object ${ }^{16}$, «la même signification-stimulus », ou plus précisément le même "parcours stimulatoire», ou encore le même neural intake, pour reprendre une des dernières expressions utilisées par Quine ${ }^{17}$ pour exprimer cette idée de l'excitation des récepteurs transmise jusqu'aux neurones à laquelle correspond tel ou tel énoncé d'observation.

Le résultat de l'analyse logique sur laquelle se développe l'épistémologie naturalisée est un empirisme éclairé ou externalisé (selon l'expression de Quine ${ }^{18}$ ) car il se distingue par l'abandon de l'introspection encouragée par l'empirisme 
traditionnel. On ne s'attache plus à établir le lien entre sensation et réflexion, ou entre impressions et idées, mais plutôt entre énoncés d'observation et stimulations des récepteurs nerveux d'un côté, et énoncés d'observations et énoncés théoriques de l'autre ${ }^{19}$.

Pourquoi l'empirisme a-t-il progressé selon Quine en se réformant de la sorte? Une lecture attentive de «L'épistémologie devenue naturelle 20 » montre que cette question est cruciale pour comprendre et évaluer le système de Quine: saisir en quoi l'empirisme réformé réalise un progrès selon Quine, c'est aussi voir la portée du behaviorisme, de la thèse de l'indétermination de la traduction, ainsi que celle de l'absence de frontière nette entre l'analytique et le synthétique. Accorder ou contester ce progrès de l'empirisme réformé, c'est en quelque sorte juger de la réussite ou l'échec du système de Quine. Mais je laisserai ce second point aux débats philosophiques; il faut comprendre avant de juger.

Le point décisif, souligné par Gibson, est que la science de la nature et l'empirisme se contiennent mutuellement, en un sens qui mérite d'être précisé21. La science nous apprend deux principes fondamentaux de l'empirisme qui restent pour Quine hors de contestation: «L'un est que toute preuve qu'il peut y avoir pour la science est d'ordre sensoriel. L'autre [...] est que toute injection de signification dans les mots doit en fin de compte reposer sur des preuves sensorielles22.» Mais, pour Quine, la science et la philosophie ont aussi conduit l'empirisme à deux abandons majeurs: celui de l'espoir de dériver les vérités des sciences de la nature à partir des données sensorielles (impasse rencontrée par Hume), et celui de traduire ces mêmes vérités en termes sensoriels (échec de l'Aufbau de Carnap). Il est impossible de développer ici les raisons de ces deux abandons. Je vais donc insister uniquement sur les arguments en leur faveur.

Le premier argument est que toute généralisation sur le monde sensible est irréductible à un quelconque ensemble de preuves sensorielles car un énoncé universel sur le monde sensible contient plus de cas que l'on ne peut en observer. 
Nous ne savons jamais par l'observation qu'un énoncé catégorique d'observation, comme

(1) «Quand un saule croît au bord de l'eau, il penche sur l'eau »

est vrai. L'observation pourrait, comme l'a martelé Popper, infirmer l'énoncé, mais jamais le confirmer. Il s'ensuit que les énoncés catégoriques d'observation constituent les points de contrôle empirique des théories ${ }^{23}$, même si le contact ultime de tout langage avec le monde se manifeste via les énoncés d'observations. En effet, seuls les énoncés catégoriques d'observation se prêtent, grâce à leur caractère permanent, à l'implication au sein d'une théorie scientifique ${ }^{24}$.

Le fait que les énoncés catégoriques d'observation soient considérés par Quine comme le contenu empirique contrôlable d'une théorie, à la différence des énoncés occasionnels d'observation qui, de par leur indifférence à la structure logique de la théorie, restent impropres ce rôle, appelle deux remarques.

La première est que l'empirisme, comme Quine le rappelle à Davidson, n'est pas une théorie de la vérité, mais une théorie de la croyance justifiée et que celle-ci repose à la fois sur l'évidence sensorielle et la logique ${ }^{25}$. L'empirisme ainsi compris a un aspect à la fois descriptif et normatif et l'on verra pourquoi plus loin il n'est pas un dogme pour Quine, mais une thèse à laquelle il est improbable que l'on puisse raisonnablement renoncer.

La seconde remarque porte sur la justification mutuelle de l'abandon de l'espoir de dériver les vérités de la science à partir des données sensorielles, et celle du holisme modéré. Si le contenu empirique d'une théorie réside dans les énoncés catégoriques d'observation, ce sont des couples d'observations (et jamais une observation isolée) qui peuvent infirmer une théorie: dans l'exemple des saules, c'est l'observation d'un saule au bord de l'eau mais penchant du côté opposé au point d'eau qui infirmerait l'énoncé catégorique d'observation. Or, à supposer que l'énoncé (1) soit réfuté par le couple d'observations en question, sa fausseté ne réfute 
jamais une hypothèse isolée de la théorie scientifique, comme celle par exemple que les racines d'un arbre nourrissent surtout le côté de l'arbre où celles-ci se trouvent, mais aussi que les racines reçoivent d'avantage de nourriture d'un terrain plus humide, etc. Ce qui est réfuté, comme la remarqué Duhem, c'est la conjonction des hypothèses dont on a eu besoin pour impliquer l'énoncé catégorique d'observation. Le holisme modéré prétend seulement que le savant doit alors supprimer au moins un énoncé parmi cette conjonction d'énoncés théoriques qui impliquent l'énoncé catégorique faux, en obéissant à la devise de la mutilation minimum et de la maximisation de la simplicité, de façon à maximiser le succès futur des prévisions: expliquer, dans l'exemple choisi dans quelles conditions (1) est vrai et dans lesquelles il est faux. Le holisme ainsi entendu a une conséquence remarquable: il est faux que chaque énoncé scientifique ait son propre contenu empirique isolable; il y a partage du contenu empirique parmi les énoncés d'une théorie scientifique, et même, insiste Quine, dans les mathématiques appliquées ${ }^{26}$. On a insuffisamment aperçu que le holisme modéré établit le lien via l'épistémologie, avec la thèse de l'indétermination de la traduction ou plus exactement avec celle de la sous-détermination empirique des théories ${ }^{27}$. Cela ne signifie pas qu'il faille abandonner l'idée selon laquelle la signification d'un énoncé réside dans la vérité ou la fausseté de celui-ci pour l'expérience possible, mais que l'on est contraint d'assainir cette théorie vérificationniste de la signification en admettant que l'évidence sensorielle ne se répartit pas sur des énoncés indépendants, encore moins sur des termes, mais sur notre théorie tout entière 28 .

Cette indétermination de la traduction mène aisément au second abandon décisif pour la naturalisation de l'épistémologie: celui de l'espoir d'une traduction (ou d'un réduction) des vérités des sciences de la nature exprimée en termes observationnels et logico-mathématiques. Une telle traduction aurait idéalement montré le caractère innocent et superflu du vocabulaire physicaliste (qui consiste à parler en 
termes de «corps») de notre langage ordinaire et de nos théories physiques. Or la sous-détermination empirique des théories scientifiques interdit l'accès à une quelconque clé de traduction des énoncés physicalistes dans un langage où le vocabulaire des phénomènes et de la théorie des ensembles définit et donc élimine le vocabulaire des corps. Une telle relation d'équivalence n'existe pas parce que c'est l'ensemble des énoncés de nos théories qui sont reliés à notre expérience sensorielle, et non les énoncés théoriques ou les termes pris isolément ${ }^{29}$.

Dès lors que le projet d'une reconstruction rationnelle du langage sur la nature et abandonné, il ne s'agit pas d'abandonner l'épistémologie comme on l'a fait pour la métaphysique, mais plutôt de considérer celle-ci comme «un chapitre

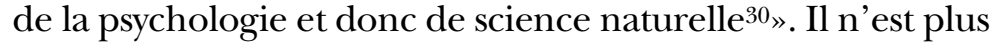
de mise de s'inquiéter de la circularité qu'il y avait, dans l'ancienne épistémologie, à vouloir expliquer les sciences de la nature en se fondant sur une science qui appartient elle aussi à ce groupe. L'abandon du vieux rêve d'une philosophie première va de pair avec l'usage de toutes les ressources scientifiques disponibles ${ }^{31}$ pour expliquer la genèse de la science. Ainsi le concept physique d'excitation des récepteurs sensoriels peut légitimement être utilisé dans l'explication du processus causal des énoncés d'observation, et «la conscience interviendra où elle voudra's2». Nul besoin d'un recours à l'introspection ou d'un quelconque privilège accordé à l'expérience du sujet pour expliquer comment la science se développe et s'apprend. Une fois naturalisée, l'épistémologie ne disparaît pas, mais son entreprise est une entreprise scientifique comme une autre. Si on la soupçonne à tort de n'avoir plus rien à faire dès lors avec la philosophie, c'est peut-être parce qu'elle donne des solutions à des problèmes classiques de philosophie, en montrant par exemple que le doute sceptique, fondé sur les illusions des sens, présuppose lui-même l'acceptation de normes scientifiques sur la réalité extérieure. Ce point est d'importance parce qu'il montre le lien de la naturalisation de l'épistémologie et de la conviction qu'a tou- 
jours eu Quine au sujet du scepticisme : les vrais doutes sont ceux que soulèvent les sciences et le scepticisme absolu ou transcendant est absurde ${ }^{33}$.

Je n'évoquerai pas toutes les critiques que la théorie de la naturalisation de l'épistémologie a provoquées ${ }^{34}$. La première qui peut venir à l'esprit d'un lecteur possédant au moins un vernis de culture philosophique serait probablement de considérer que l'épistémologie de Quine est un scientisme, avec la nuance péjorative qu'a ce terme lorsqu'il désigne une confiance dogmatique et naïve dans les enseignements de la science. Il faut avec Gibson accepter, à défaut d'un autre terme, celui de scientisme, mais tenter de laver celui-ci de son acception péjorative.

La thèse de la sous-détermination empirique des théories, de l'indétermination de la traduction, suffit à lever l'accusation de naïveté. Quant au risque pernicieux de dogmatisme, on rappellera qu'aucune théorie, qu'aucune vérité, fut-elle mathématique, n'est susceptible d'être immunisée contre une révision. L'empirisme n'échappe pas à la règle : il pourrait «filer par dessus bord» si des données factuelles extraordinaires venaient accréditer la télépathie et la voyance. Il faudrait alors admettre la prédiction d' "entrées " télépathiques et divines aussi bien que sensorielles ${ }^{35}$. Une telle supposition, ajoute Quine, est futile au regard du manque de plausibilité de pareils événements. Il y a donc bien quelque chose de déraisonnable dans le rejet métaphysique de l'empirisme réformé et normatif.

\section{La reconstruction behavioriste de la signification}

L'analyse que Quine fait de la signification est faite dans un esprit naturaliste et ce naturalisme entraine une théorie behavioriste (comportementaliste) de la signification. Celle-ci s'appuie principalement sur l'observation de l'apprentissage du langage. Il est remarquable que Quine définisse le langage par son mode d'acquisition:

Le langage est un art social que nous acquérons tous unique- 
ment en reconnaissant le comportement manifeste d'autrui lors de circonstances publiquement identifiables ${ }^{36}$.

Le behavorisme de Quine est une expression de son empirisme : la connaissance scientifique du monde repose sur des données observables dont l'une, quasi constante, est le comportement de nos semblables. Word and Object paraît en 1960, deux ans après l'ouvrage au titre explicitement behavioriste, auquel Quine fait référence : Science and Human Behavior par le psychologue Skinner. Le behaviorisme semble être depuis un peu passé de mode en psychologie, mais c'est surtout dans le domaine de la linguistique que Quine en fait usage sous le poids d'une nécessité qu'il estime aussi incontournable que naturelle:

Je tiens en outre la voie d'approche behavioriste pour obligatoire. En psychologie, on peut ou non être behavioriste, mais en linguistique le choix n'existe pas. Chacun de nous apprend sa langue en observant le comportement verbal des autres et en voyant les hésitations de son propre comportement observées et encouragées ou corrigées par les autres ${ }^{37}$.

Cependant, au-delà de cette insistance sur l'apprentissage du langage, ce n'est que pour répondre à des critiques, notamment celles de Chomsky38, que Quine a donné quelques précisions sur ce qu'il entend par behaviorisme, afin d'éviter les contresens. Parmi ceux-ci, la tendance à l'opposer au rationalisme, en arguant du caractère inné de certaines structures cognitives contre la thèse selon laquelle toute la connaissance dériverait de l'expérience. Or Quine adopte à la fois l'innéisme et le rationalisme et rejette cette version maladroite de l'empirisme. Si le behaviorisme prête une telle attention aux processus de renforcement et d'extinction des réponses pour comprendre l'apprentissage, c'est qu'il doit aussi admettre que ces réactions dépendent de dispositions non apprises et donc innées ${ }^{39}$. Par exemple, le rapprochement sous un même terme d'objets qui ont une couleur semblable dépend d'une norme innée de similarité, et l'affirmation de l'existence d'une telle norme n'est pas anti-empiriste, mais reste un lieu 
commun de la psychologie "véridiquement interprétable en termes de comportement ${ }^{40 » \text {. }}$

La théorie behavioriste de la signification peut se résumer une thèse négative - les significations ne sont pas des entités indépendantes du langage, - et une thèse positive selon laquelle la signification peut se définir par l'usage pertinent des expressions d'une langue. Parce qu'elle nécessite le recours à la logique formelle, on laissera l'étude de la thèse négative pour la dernière section. Après avoir examiné la thèse positive, on exposera deux thèses célèbres de Quine qui sont des conséquences de son holisme et de son behaviorisme: la première est la critique de la distinction de l'analytique et du synthétique, la seconde est l'indétermination de la traduction.

En répudiant les propositions ou les contenus intensionnels ${ }^{41}$ de son ontologie, Quine s'attaque à la théorie du langage-copie, entretenue par une sémantique non critique et par la pensée commune. Cette théorie se résume au «mythe d'un musée où les articles exposés sont les significations, et les étiquettes les mots ${ }^{42}$.» L'observation du langage, aussi bien dans la phase d'apprentissage que dans celle du perfectionnement, suffit à montrer que ce mythe du langage musée est un mythe pernicieux qui s'oppose à une compréhension correcte des phénomènes linguistiques. Car cette observation ne nous autorise pas à croire qu'il existe quoi que ce soit que l'on puisse dire être des significations, quelle que soit la façon dont on les définit (images mentales, idées platoniciennes, etc.).

Le phénomène d'échange linguistique est une interaction entre des individus. Celle-ci va des premiers mots du nourrisson jusqu'aux productions les plus sophistiquées de la science et de la littérature. Quelle signification a le mot signification lorsqu'il désigne quelque chose que ces échanges linguistiques auraient en partage? Il est difficile de le dire puisqu'il y a un monde entre apprendre, par ostension, à désigner les objets concrets, et comprendre ce que décrivent les énoncés les plus abstraits des sciences exactes. Mais avant d'aller plus loin il faut insister sur le fait qu'il n'y a pas pour 
Quine quelque chose qui soit la signification, et que l'idée de l'existence d'une signification transcendante à la communication linguistique proprement dite est à ses yeux une illusion dont il faut se débarrasser.

Pour éclairer la position de Quine au sujet de la signification, je vais me risquer à reprendre un exemple utilisé par Russell, en précisant plus loin le point de désaccord qui peut subsister, dans l'analyse de cet exemple, entre Quine et Russell. Les philosophes de la tradition analytique (au sens large) reconnaissent d'une façon générale que la voie la plus simple pour définir ce que l'on entend par la signification d'une expression, ainsi que pour la distinguer de sa référence, est de prêter attention à l'usage des guillemets. Dans Signification et Vérité, Russell remarque qu'il y a trois manières pour une phrase d'apparaître dans un contexte citationnel ${ }^{43}$. La première est la citation au style direct, qui ne présuppose pas que l'on comprenne la langue utilisée dans la phrase citée. Tel est le cas si je rappelle que César a dit: «alea jacta est». La seconde est le style indirect où je peux traduire le sens de ce qu'a voulu dire la personne que je cite, en affirmant par exemple que César a dit que les dés sont jetés. La troisième se rapprocherait de ce que les grammairiens appellent le discours indirect libre, où l'on asserte ce qui a été dit par le locuteur: «Les dés furent jetés, comme le disait vraiment César.»

Puisqu'il s'agit d'analyser la signification, laissons de côté le premier usage de la citation, où les mots sont cités en tant que mots, non pas en tant qu'ils ont un sens. Attardons-nous sur les deux autres usages de la citation. Il est intéressant d'avoir ici affaire à une phrase citée qui est une métaphore, car il est vrai que Quine a peu développé l'analyse logicophilosophique des métaphores, ce qui ne signifie pas que sa théorie soit incapable d'en rendre compte. Lorsqu'on rappelle que César a dit que les dés sont jetés, il est certain que c'est le sens métaphorique de l'expression «les dés sont jetés» qui importe, car, comme le souligne Russell avec humour, il est hors de question de savoir si en fait, des dés furent ou non jetés. L'identité de signification entre «alea 
jacta est» et «les dés sont jetés » réside dans la traduction d'une expression par l'autre et par le fait que les individus maîtrisant les deux langues approuvent le passage du style direct au style indirect tel qu'il est donné dans l'exemple. Cependant, ce qui rend ici l'analyse plus difficile est le fait que nous n'avons pas affaire à un énoncé d'observation comme: "voilà un chat », mais à un usage abstrait et sophistiqué du langage, et l'on doit supposer l'équivalence de groupes d'énoncés entre les deux langues pour qu'une telle métaphore puisse être interprétée avec succès. On est loin de la stimulation nerveuse isolable et concomitante à l'énoncé observationnel, mais on est simplement engagé plus profondément dans la complexité du réseau linguistique, ce qui ne signifie nullement que l'on a quitté le sensible pour entrer dans le royaume des Idées. Ici l'entreprise de traduction, c'est-à-dire d'interprétation, réussit; en revanche, tenter une traduction des traités de la mécanique quantique dans le latin de Cicéron est une entreprise absurde et vouée à l'échec. La question de la signification d'un énoncé ne peut donc pas se résoudre sans que l'on s'interroge sur l'identité de signification avec un autre énoncé. Qu'interviennent alors la psychologie, la physiologie, et d'autres sciences pour contribuer à l'analyse des significations montre simplement la cohérence de la position de Quine.

La phrase citée peut aussi être assertée par celui qui la cite, alors elle indique quelque chose, et elle est fausse, si elle n'indique rien. Quine s'accorde avec l'idée largement développée par Davidson, selon laquelle le concept de vérité est à la fois primitif et central et que c'est à partir de lui que l'on peut analyser le concept de signification: comprendre la signification d'une phrase, ce n'est pas être capable de dire si cette phrase est vraie, mais c'est être en mesure de décrire ou d'expliquer ce qui la rendrait vraie. On pourrait objecter que cette définition de la signification via la référence souffre d'une trop grande généralité. Mais l'objection ne tient pas: il est naturel que la compréhension d'une expression doive se tester à l'aide d'autres énoncés qui appartiennent à notre 
bagage linguistique et à notre compréhension globale du monde: «comprendre, du point de vue du comportement, est donc un effet statistique, résidant dans la multiplicité 44 ».

Quine se démarque cependant de l'analyse que Russell donne de la signification dans An Inquiry, sur des points qui ne sont pas de simples détails. La première différence sur laquelle il convient d'insister est que, même si Russell parvient à définir les propositions, c'est-à-dire les significations, comme des réalités non-verbales, comme des «occurrences psychologiques - images complexes, attentes, etc.», cette analyse de la signification est débarrassée de tout contenu mentaliste chez Quine. On ne peut pas effet établir une équivalence entre les stimuli auxquels Quine fait référence par son expression de signification-stimulus et les images ou les attentes dont il est question dans l'analyse russellienne. Au fait de la psychologie expérimentale, Quine sait que ce qui constitue un stimulus peut ne pas être un événement psychologique, mais peut être inféré du comportement, tout comme d'ailleurs les images mentales. Pour un énoncé observationnel, l'identité des significations a sa source dans l'accord des parcours stimulatoires. Pour des énoncés qui ne sont pas observationnels, mais qui sont occasionnels, l'identité de signification peut être testée par la cohérence du comportement verbal du locuteur: si le célibataire est un homme non marié, là où il est acquiesce à un énoncé au sujet des célibataires, il doit aussi acquiescer à ces mêmes énoncés où l'on aura substitué «individus non mariés » à «célibataires».

Cependant la substituabilité des expressions synonymes ne constitue pas un critère infaillible de l'identité en signification, non parce qu'il n'existe pas d'expressions au sujet desquelles on puisse dire qu'elles sont synonymes, mais tout simplement parce qu'il n'existe aucune identité des significations qui soit telle qu'elle puisse passer toujours sans dommage tous les tests que l'on puisse imaginer dans toutes les situations concevables. Si cela était le cas, alors il faudrait réhabiliter le mythe du musée des significations, tout comme le caractère absolu de la distinction de l'analytique et du syn- 
thétique s'imposerait, et la thèse de l'indétermination de la traduction s'effondrerait. Or Quine montre qu'il n'y a aucune possibilité de définir rigoureusement l'analycité sans circularité, et que la thèse de l'indétermination de la traduction (ou de la référence), au moins dans sa version faible, s'impose comme une vérité de la logique contre laquelle il serait vain de s'acharner. Examinons ces deux arguments.

Quine remarque lui-même que «célibataire » et «homme non-marié » ne sont pas rigoureusement synonymes, puisque l'on peut objecter le cas des personnes divorcées ou celles qui étant encore dans l'enfance, ne sont pas mariées, sans qu'il soit vraiment pertinent de dire qu'elles sont célibataires ${ }^{45}$. On se trouve donc dans un cas analogue à celui que Quine signale ailleurs: même si tous les animaux avec un cœur sont aussi des animaux avec des reins, autrement dit même si «animal avec un cœur » et "animal avec des reins", ont la même extension, il n'est cependant pas douteux que l'on refusera d'accorder une quelconque synonymie à ces deux expressions ${ }^{46}$. Le cas de «célibataire » et «individu non marié » est comparable. Je ne peux pas établir a priori que les deux expressions sont substituables dans tous les contextes référentiellement transparents car, comme le montre la restriction au sujet des divorcés et des enfants, la synonymie des deux expressions peut susciter des objections. Pour corriger ce défaut, on aura recours au dictionnaire pour préciser la définition de célibataire par «individu en âge d'être marié et ne l'étant pas ", mais on peut parier que l'on trouvera indéfiniment des contextes purement référentiels où l'usage du terme «célibataire » résistera au test de la substituabilité (que l'on songe à des phrases françaises où il est question de "vieux célibataires », où la signification de l'adjectif vieux n'est donnée que par le contexte). La leçon à tirer de cette situation est qu'il n'existe pas de définition absolument générale de l'analycité qui soit capable de nous sortir d'embarras: pour dire que l'énoncé «tout célibataire est un individu en âge d'être marié mais non marié » est un énoncé analytique et dont l'analycité est plus évidente que «tout céliba- 
taire est un individu non marié », il faut avoir une compréhension préalable de ce que signifie la notion d'analyticité. Des philosophes protesteront contre l'inadéquation de l'exemple: «être en âge d'être marié » est une clause qui varie selon les sociétés et les époques et il y a peu de chances d'obtenir une définition claire de l'analycité en partant d'exemples aussi contestables.

Pourtant le holisme non seulement autorise mais justifie pleinement un recours à ce type d'exemple qui au contraire se montre plus éclairant qu'on ne le pense. Lorsque Kant considère que «tout corps est étendu » est un jugement analytique, il hérite de la théorie cartésienne et galiléenne des corps. Analytique dans la théorie cartésienne, cet énoncé l'est-il pour tout être pensant? Et qu'en est-il de l'énoncé einsteinien «tout corps est une quantité d'énergie »? Ces hésitations sont l'indice pour Quine qu'il n'y a pas de différence nette entre faits et théories et qu'il est inutile que l'empirisme conserve cette relique héritée de la métaphysique essentialiste: la philosophie du langage de l'épistémologie naturelle nous montre qu'il faut renoncer à chercher les significations (éternelles) des mots de la même façon que l'évolutionnisme nous débarrasse d'une croyance en l'essence des êtres. L'analycité entretenait cette croyance. Il était important que Quine allège cette notion de son fardeau métaphysique ${ }^{47}$.

Si c'est le holisme qui explique plus directement la thèse, contre le caractère absolu de la distinction de l'analytique et du synthétique, c'est le behaviorisme qui a pour conséquence, ainsi que Quine le reconnaît explicitement, la fameuse thèse de l'indétermination de la traduction. Cette thèse qui a fait couler beaucoup d'encre a souvent été mal comprise. Pour dissiper les malentendus à son sujet, il est nécessaire de comprendre que si cette thèse est une des conséquences du behaviorisme de Quine, celle-ci n'implique aucunement, comme on le verra plus loin, un scepticisme philosophique sur la capacité des théories scientifiques à atteindre la vérité. 
La version faible de l'indétermination de la traduction qui concerne les termes des énoncés et non les énoncés pris comme totalités - la version que Quine a baptisé «l'inscrutabilité de la référence» (ou, plus récemment, «indétermination de la référence ») - est en réalité une thèse «triviale et indiscutable ", comme Quine l'affirme un peu sèchement ${ }^{48}$. La version forte, l'indétermination de la traduction proprement dite, s'applique aux énoncés pris holophrastiquement (c'est-à-dire formant un tout): elle reste une thèse qui, au contraire, conserve un caractère entièrement spéculatif que Quine n'a pas vraiment cherché à nier. Quine résume ainsi comment il est parvenu à cette thèse si débattue qu'est l'indétermination de la traduction:

La signification d'un énoncé dans une langue est ce qu'il partage avec ses traductions dans une autre langue - en raison de quoi j'ai proposé mon expérience de pensée de la traduction radicale. Elle a conduit à une conclusion négative, à la thèse de l'indétermination de la traduction ${ }^{49}$.

L'expérience de pensée de la traduction radicale consiste à supposer un linguiste en contact avec une tribu dont il ignore complètement le langage. Les seules données qu'il dispose pour établir son manuel de traduction sont les émissions verbales des indigènes lors des circonstances qu'il partage avec eux. La première conclusion à laquelle Quine parvient est que la référence est inscrutable, au sens où il n'existe aucune donnée empirique qui soit capable de nous permettre de trancher entre différentes traductions possibles d'un énoncé de la langue indigène prononcé pour désigner quelque chose par ostension. Le célèbre "gavagai", pris en exemple comme l'énoncé dont l'indigène fait usage pour désigner le lapin, peut être traduit aussi bien par «lapin», "partie non détachée de lapin», "segment temporel de lapin », ou encore «image de lapinité50». Ce qui est «empiriquement significatif» c'est le næud ou l'objet auquel renvoient ces expressions, qui ne diffèrent pas seulement du point de vue intensionnel,mais diffèrent aussi selon du point de vue de 
leur extension: «lapin », «partie non détachée de lapin» et «segment temporel de lapin» contiennent des termes qui ne mobilisent pas la même ontologie ${ }^{51}$. La référence à une partie non détachée de lapin enveloppe une référence à une multiplicité de parties, ce que ne fait pas l'énoncé ordinaire «voilà un lapin ». De la même façon, une description purement événementielle du monde requière une quantification sur les instants à laquelle la description objectuelle n'est pas immédiatement contrainte. Il n'est pas non plus égal, d'un point de vue ontologique, de parler du vert comme terme général vrai de tous les objets verts, et du parler de la couleur qu'est le vert. Or désigner une chose verte en disant «vert» laisse indéterminée la référence et aucune donnée empirique non verbale ne semble en mesure de dépasser cette indétermination.

Les linguistes peuvent trouver que l'argument et les exemples de Quine sont artificiels: le traducteur optera pour la simplicité et le naturel dans la traduction, et il traduira «gavagai » par «lapin », ou encore «voici un lapin». Il écartera sans même y songer les traductions farfelues comme "partie non-séparée de lapin» ou «segment temporel de lapin ». Mais Quine entend traiter d'un point de philosophie qui porte sur l'ontologie et non sur les problèmes de traduction en tant que tels.

L'indétermination de la référence est à la fois incontestable et inoffensive. En réalité, elle est surmontée par les interprètes qui prennent appui sur le contexte et portent attention à la simplicité, aux niveaux de langues et à tous les éléments qui, lorsque l'interprète a du talent, font oublier que l'on a affaire à une traduction. Mais cela confirme encore l'idée de Quine selon laquelle «le traducteur radical doit imposer à peu près autant qu'il découvre». Les situations linguistiques imaginées par Quine montrent que l'ontologie d'une théorie devient indifférente dès lors qu'il est possible de traduire les expressions de la langue étrangère ou du manuel de traduction rival de façon à être toujours à portée de ce qui est désigné ou dénoté dans le lexique de la 
langue traduite. Quine soutient que «la seule chose empiriquement significative dans une ontologie, sont les nœuds, par eux-mêmes neutres, qu'elle procure à la structure de la théorie ${ }^{52}$.» Il importe peu que l'on fasse référence à tel corps en le désignant comme un individu simple ou comme l'ensemble de ses parties ou encore comme l'univers moins le corps lui-même, pourvu que l'on puisse reconnaître les énoncés où ce corps fait l'objet d'une quantification existentielle, norme ultime de l'engagement ontologique, ainsi qu' on le verra dans la section suivante.

La thèse forte de l'indétermination de la traduction ne porte pas sur les termes des énoncés, mais sur les énoncés eux-mêmes, incompatibles d'un manuel de traduction à l'autre, ou d'une théorie à l'autre ${ }^{53}$. En généralisant la thèse de l'indétermination de la référence, Quine maintient que l'indétermination affecte le passage d'une langue à une autre, ou, à l'intérieur d'une même langue, d'un manuel de traduction rival à un autre, ou encore, d'une théorie scientifique à une théorie rivale. Cette thèse forte, beaucoup plus controversée, est imposée par le holisme. Néanmoins, comme Quine le reconnaît, il n'est guère aisé d'illustrer la thèse forte de l'indétermination de la traduction. Une des raisons, comme le souligne Gochet, est que ce ne sont pas les langages qui font l'objet de traductions, mais les séquences d'énoncés 54 .

Du seul fait que l'indétermination de la traduction est difficile à illustrer, puisque l'on ne compare jamais vraiment des langages dans leur globalité, pourquoi Quine maintientil cette thèse qu'il est difficile de ne pas soupçonner d'être à la fois sceptique et métaphysique? La première raison est que, malgré tout, l'indétermination de la traduction peut être illustrée à l'aide de théories scientifiques logiquement incompatibles, mais empiriquement équivalentes, comme les géométrie de Riemann et d'Euclide. C'est à ce sujet que Quine reprend un exemple donné par Poincaré ${ }^{55}$. Or Quine n'a jamais abandonné la thèse d'une absence de rupture entre le langage ordinaire et les théories scientifiques où les 
énoncés d'observation sont, de manière analogue, le point de départ et la pierre de touche de la vérification.

L'indétermination de la traduction conduit donc à rejeter la métaphysique des propositions comme significations des énoncés, mais elle préserve aussi du dogmatisme, via le corollaire de la sous-détermination empirique des théories, puisqu'elle montre que plusieurs théories du monde sont concevables. Reconnaître la pluralité des théories et le fait que celles-ci imposent autant qu'elles ne découvrent, ce n'est pas apporter de l'eau au moulin du scepticisme. Nous allons voir pourquoi la vérité est aussi indispensable pour une compréhension correcte de ce qu'est l'engagement ontologique d'une théorie.

\section{Logique et Ontologie}

La logique apporte une simplification et une clarification du schème conceptuel de la science. Infiltrée dans toutes les branches de la connaissance, tous les progrès qu'elle réalisent intéressent la philosophie. Or Quine a eu le mérite de voir que la logique classique de la quantification était capable d'apporter le critère le plus simple qui soit pour comprendre l'engagement ontologique d'une théorie. Plus précisément, une théorie quelconque admettra des objets d'une certaine sorte, si et seulement si ces objets doivent appartenir aux valeurs des variables qui rendent la théorie vraie. D'où le slogan, inlassablement répété par Quine: «être, c'est être la valeur d'une variable». Autrement dit, il y a des choses de l'espèce $F$, si et seulement si l'énoncé $F x$ est vrai, c'est-à-dire, si et seulement si il existe au moins un individu qui est vrai de $F$. La simplicité de ce critère, exprimable dès que l'on est en possession de la théorie classique des prédicats du premier ordre, est telle que qu'on peut l'appliquer à l'ensemble des théories qui conservent les vérités et les procédures de la logique classique de la quantification.

Le critère d'engagement ontologique ne dit pas ce qui existe. Il permet de dire ce qu'une théorie assume comme 
existant. Son mérite est de rendre explicite les engagements cachés et clarifie les disputes ontologiques. Il permet par exemple de montrer que le Russell des Principia Mathematica accepte l'existence des classes à son insu, tout comme il jette une lumière sur les difficultés de la prétention nominaliste à faire l'économie des objets abstraits.

Ce critère n'est pas non plus l'expression d'une conception purement linguistique de l'ontologie. Le fait d'être ou d'exister pour un corps n'est pas quelque chose de verbal. L'information au sujet de ce qui existe repose toujours d'une façon ou d'une autre, d'une manière plus ou moins complexe, sur l'évidence sensorielle. C'est cependant à l'aide du langage ou de nos théories que nous affirmons ce qui existe et c'est pourquoi l'usage du critère d'engagement ontologique se révèle crucial: il permet de distinguer les engagements ontologiques des théories, et ce n'est que lorsque ceux-ci sont aperçus clairement que l'on peut discuter de leurs justifications respectives.

Une autre idée fausse au sujet de l'engagement ontologique serait d'imaginer que ce critère ne porte que sur l'existence et qu'il laisse de côté la réflexion sur l'être, objet véritable de la spéculation philosophique auquel Quine, en tant qu'épistémologue, ne s'est pas vraiment attaqué. Or pour Quine s'exprimer sur l'être en général, indépendamment d'une théorie scientifique définie sur tel ou tel domaine de la connaissance, n'a que très peu d'intérêt philosophique. Etre, c'est toujours être quelque chose, avoir une ou des propriétés définies, comme l'énoncent clairement les énoncés existentiels dans un langage du premier ordre. Les objets qui existent sont les valeurs qui satisfont les énoncés où figurent les variables de la quantification. Etre, c'est être la valeur d'une variable.

S'il n'y a pas de sens à parler de l'être en général, il est tout aussi vain de vouloir tracer une frontière, comme le fait Carnap, entre les questions ontologiques et les questions empiriques. Certes, les questions d'existence en mathématiques peuvent être envisagées comme relevant du choix d'un 
langage, mais les hypothèses de physique sont elles aussi dépendantes de choix théoriques ${ }^{56}$. Pas plus qu'il n'y a de frontière strictement définie entre l'analytique et le synthétique, il n'y en a entre l'ontologique et l'empirique ou entre les énoncés de fait et les énoncés théoriques. La mathématique, dans la région des théories des ensembles, semble donner du poids au conventionalisme, mais il n'en subsiste pas moins que le critère d'engagement ontologique s'y applique avec la même rigueur et que l'on peut comparer les univers des divers systèmes comme on compare les populations des régions. La simplicité de ce critère s'accorde sans difficulté avec la conception holiste de la science.

Quine admet cependant que le critère d'engagement ontologique souffre d'un inévitable «esprit de clocher »: la quantification existentielle qui est au principe du critère d'engagement ontologique est une quantification standard, c'est-à-dire objectuelle, et ne peut en toute rigueur s'accorder avec la théorie intuitionniste de la quantification. Les restrictions intuitionnistes conduisent en effet à n'accepter l'existence d'objets mathématiques que si l'on est en mesure de donner des preuves directes ou des procédés de construction effective de ceux-ci. Quine répond à l'objection intuitionniste en reconnaissant sa «conception déviante de l'être » qui le conduit à une ontologie différente de la sienne, voire à une expression de celle-ci qui n'est peut-être pas traductible dans la notation standard ${ }^{57}$.

L'usage de la logique dans le système philosophique de Quine doit être compris dans un esprit naturaliste. Si, parmi les outils de l'analyse philosophique, Quine privilégie la logique propositionnelle classique (mieux nommée pour Quine «logique ou calcul des énoncés»), ainsi que la théorie classique des prédicats du premier ordre, c'est que cette logique jouit des propriétés d'évidence et de simplicité. Elle est au cour de notre compréhension du monde et l'on ne peut y renoncer sans bouleverser profondément le système de nos connaissances. C'est d'ailleurs sur ce type de considérations naturalistes et holistes que Quine explique la «néces- 
sité mathématique »: il est plus raisonnable de rendre compte de celle-ci par notre volonté de «ne pas trop faire chavirer le navire », pour reprendre ses mots, que d'attribuer aux énoncés mathématiques une nécessité éternelle. Mais le calcul des énoncés ainsi que le calcul des prédicats sont la logique au sens strict ou restreint. Celle-ci se distingue des mathématiques en ce que la logique en ce qu'elle est ontologiquement neutre, c'està-dire qu'elle ne suppose pas l'existence d'un type défini d'objet, mais au contraire peut accueillir tout ce qui peut être considéré comme un objet. Tel n'est pas le cas par exemple de la théorie des ensembles classique, qui comporte des distinctions ontologiques (éléments, ensembles, ensembles de parties), et des postulats existentiels comme l'axiome de l'infini ainsi que des axiomes ad hoc, comme celui de séparation, dont la fonction est d'éviter les antinomies ensemblistes. Rien de semblable dans la logique proprement dite. Celle-ci joue son rôle dans les théories des ensembles rivales, mais ne se confond avec aucune d'entre elles.

On comprend mieux alors l'usage parfois déroutant que Quine fait de démonstrations logiques extrêmement simples afin de montrer les raisons pour lesquelles il est philosophiquement douteux de considérer les pensées ou les propositions comme des entités. Si celles-ci pouvaient être considérées comme des objets, les termes qui désignent ces mêmes objets devraient pouvoir être substituables l'un à l'autre salva veritate dans un discours purement référentiel. Or les énoncés qui servent à indiquer les croyances ou les pensées en général sont référentiellement opaques, c'est-à-dire mettent en échec l'extensionalité58. La propriété de substituabilité des termes co-désignatifs est une propriété commune des théories extensionnelles qui sont dites référentiellement transparentes. Les constructions où apparaissent des attitudes propositionnelles, c'est-à-dire la référence aux croyances ou aux pensées, montrent l'opacité de la référence par l'échec de la substituabilité des termes co-désignatifs. On ne donnera que l'exemple suivant, emprunté à Quine ${ }^{59}$ : 
(1) Philippe croit que Tegucigalpa est au Nicaragua.

On admet que Philippe croit vraiment ceci et donc que (1) est vrai. Tegucigalpa est la capitale du Honduras. Il n'est donc pas possible de substituer «la capitale du Honduras» à «Tegucigalpa» sans transformer (1) en l'énoncé faux suivant:

(2) Philippe croit que la capitale du Honduras est au Nicaragua.

Il existe en fait deux façon de lire (2). La première, et la moins naturelle, est de considérer qu'il existe quelque chose que Philippe croit être au Nicaragua et qui est Tegucigalpa. Dès lors, comme Tegucigalpa est la capitale du Honduras, on peut conclure que Philippe croit, évidemment à tort, que la capitale du Honduras est au Nicaragua. C'est une façon d'interpréter (1) et (2) en rendant une croyance référentiellement transparente. Dès lors, l'argument s'interprète d'une façon que l'on dit relationnelle et que l'on peut formaliser ainsi60:

$\exists x(x=$ Tegucigalpa $\wedge$ Philippe croit $x$ au Nicaragua $)$

(Tegucigalpa = la capitale du Honduras)

$\Rightarrow(\exists x) \quad(x=$ la capitale du Honduras $\wedge$ Philippe croit $x$ au Nicaragua)

Le caractère non naturel ou incorrect d'une telle traduction saute aux yeux à la lumière de la conclusion: on ne peut attribuer à Philippe la croyance en l'existence de la capitale d'un pays qui ne serait pas dans le pays en question. Quine en conclut qu'il est préférable de s'abstenir de quantifier dans les contextes d'attitudes propositionnelles ou dans les contextes modaux. La logique apporte un argument à la thèse négative de l'épistémologie naturelle: les intensions, les croyances, les pensées, ou les propositions ne doivent pas être considérées comme des objets ou des êtres. Pas d'entité sans identité.

La seconde manière de traduire (1) est de l'interpréter de manière notionnelle, ce qui respecte l'opacité de la référence. Ainsi la formule:

Philippe croit que $(\exists x)$ ( $x$ est au Nicaragua $\wedge x=$ Tegucigalpa $)$ 
suffit à montrer que l'on ne peut pas attribuer à partir d'un tel énoncé une croyance absurde ou trop fantaisiste à Philippe. Il serait en effet illégitime dans ce contexte de substituer à «Tegucigalpa » «la capitale du Honduras », car précisément Philippe ne sait pas et donc ne croit pas que Tegucigalpa soit la capitale du Honduras. Cette dernière interprétation traduit plus naturellement ce qu'exprime (1) et montre que la lecture notionnelle, par opposition à la lecture relationnelle, doit nous prévenir de quantifier dans les contextes d'attitudes propositionnelles. Le même type d'argument est à l'œuvre pour les contextes modaux.

La leçon philosophique de cet argument logique est claire: il est vain de vouloir définir les propositions, les pensées, les idées, les désirs, tout ce qui semble avoir un contenu mental quelconque, comme des entités ou des objets. De la même façon, il n'est guère raisonnable d'attribuer au nécessaire et au possible une valeur métaphysique absolue. C'est de manière contextuelle que l'on fait référence aux pensées et aux modalités. Or, lorsque l'occurrence d'un terme singulier dans un énoncé est purement désignative, la vérité de celui-ci dépend de ce que le terme désigne, non de la manière dont il désigne. Dans cet exemple, Tegucigalpa est nommée en tant qu'objet de croyance d'un individu à un moment donné, par conséquent son occurrence dans (1) n'est pas purement désignative et c'est pourquoi le contexte est dit «référentiellement opaque».

Les conséquences de cette analyse logique ont une nature polémique évidente. Tous les systèmes philosophiques qui, plus ou moins nettement, ont accordé une recevabilité aux objets intensionnels, se voient ou bien dépourvus de toute crédibilité scientifique, ou bien contraints de préciser la sémantique dont ils font usage. Car la logique commune aux sciences de la nature est une théorie du premier ordre, où la bivalence, le tiers exclu, l'extensionalité ne posent pas problème, mais apparaissent au contraire comme des principes simples et évidents. C'est dans la logique classique que notre conception physicaliste du monde s'est développée et ren- 
forcée. On ne peut sérieusement envisager l'abandon de cette logique, sans que cet abandon ne se solde par une compréhension du monde plus grande que celle qui est aujourd'hui la nôtre. On peut admettre une telle éventualité, et considérer qu'en attendant, rien ne contraint l'épistémologue à ne pas être conservateur.

L'épistémologie de Quine, on l'a vu, est fondée sur un empirisme réformé. Avec l'évidence sensorielle, la logique est l'outil privilégié pour décider de l'ontologie de nos théories. Mais qu'en est-il précisément de l'ontologie du système philosophique de Quine? Encore une fois, le critère d'engagement ontologique n'est qu'un outil pour mettre à jour l'ontologie d'une théorie: son explication ne nous livre donc pas à elle seule l'ontologie que Quine adopte. On pourrait régler la question en disant que les objets admis par les sciences de la nature sont aussi les objets de la philosophie qui n'entend pas se distinguer de la science. Nous commençons avec des objets de taille moyenne que nous percevons et désignons dans le langage ordinaire, et nous finissons avec les neutrinos.

Une telle présentation laisse cependant de côté l'un des aspects les plus importants et les plus originaux de la philosophie de Quine, je veux parler de sa philosophie des mathématiques. On l'a dit, la logique classique de la quantification du premier ordre est ontologiquement neutre, car les individus du calcul des prédicats peuvent bien être, à dire vrai, ce que l'on veut qu'ils soient. Il n'en va pas de même lorsque nous acceptons de quantifier sur des objets abstraits comme les classes ou les ensembles, en adoptant une notation qui les distingue de leurs éléments (ne serait ce que par leur place relativement au signe d'appartenance). Quine a montré et a insisté sur le fait que «l'adoption générale de variables pour les classes débouche ainsi sur une théorie dont les lois ne peuvent pas dans l'ensemble être exprimées à l'aide des niveaux antérieurs de la logique ${ }^{61 » .}$. Adopter la théorie classique des ensembles, c'est donc adopter dans son ontologie les objets abstraits que les nominalistes rejettent. Pour qu'une théorie nominaliste des mathématiques soit possible, il fau- 
drait qu'elle puisse paraphraser la théorie classique des ensembles dans un langage aussi neutre ontologiquement que celui de la théorie des prédicats du premier ordre. Après avoir cru avec Goodman en la possibilité du nominalisme, Quine s'est séparé philosophiquement de ce dernier en énonçant de sérieux doutes sur la possibilité de construire les mathématiques classiques sur des fondements nominalistes, c'est-à-dire dans une théorie qui puisse s'abstenir de quantifier sur des ensembles ou des classes. Cette prise de position cohérente avec son critère de l'engagement ontologique l'a conduit également à critiquer son maître Carnap, pour qui la quantification sur des objets abstraits est une convention linguistique innocente du point de vue ontologique. Si la pluralité des théories des ensembles donne effectivement du poids au conventionalisme mathématique, la quantification sur les objets abstraits ne peut - aux yeux de Quine - ni être évitée, ni être considérée comme relevant de la seule "syntaxe » logique du langage, puisque la logique entendue en un sens restreint n'oblige pas plus à cette quantification qu'elle n'impose de l'éviter.

Quine ne voit donc pas de distinction nette entre l'adoption des objets abstraits ou des universaux, à laquelle nous contraint l'adoption de la théorie des ensembles, et les hypothèses des sciences naturelles, qui requièrent elles aussi le recours aux nombres et aux classes, comme en témoigne aussi la physique que la science du vivant ${ }^{62}$. Le clivage entre vérités analytiques et vérités synthétiques permet à Carnap de considérer qu'admettre ou refuser les universaux n'est en dernière analyse qu'une question interne, relevant du choix d'un langage, et non une authentique question d'existence relevant d'une enquête scientifique. Quine s'oppose à son maître en insistant sur le fait que le holisme entraîne l'abandon de la distinction absolue entre vérités analytiques et vérités synthétiques et que le même esprit naturaliste impose par conséquent de considérer que les engagement ontologiques des sciences de la nature envers les objets abstraits et celui des théories ensemblistes ne se distinguent pas clairement. 
En vertu du principe selon lequel être, c'est être la valeur d'une variable, on peut donc répondre à la question qui est de savoir quelle ontologie Quine adopte: il admet les corps, ceux auxquels nous faisons référence dans le langage ordinaire; il admet aussi l'existence des corps imperceptibles à l'expérience sensorielle commune, mais que la science postule ou atteste; et enfin il admet les classes, dont l'existence est assumée par les théories scientifiques comme la physique en général ou la mathématique. Ces classes sont les valeurs des variables des théories ensemblistes ou des théories impliquant la théorie des ensembles, et si l'on peut admettre que ces objets abstraits n'appartiennent pas aux objets physiques, il n'y a pas de sens à s'interroger sur l'existence d'objets abstraits correspondant aux énoncés existentiels des théories mathématiques. L'existence ou l'inexistence de l'ensemble de tous les ensembles est par exemple rejetée comme contradictoire dans la théorie de Zermelo-Fraenkel, alors qu'elle est prouvée dans New Foundations, la théorie ensembliste de Quine ${ }^{63}$, ce qui fournit un exemple parfait de la relativité de l'ontologie. Dans son dernier ouvrage, Quine a soulevé le problème qui est de savoir comment un empiriste peut interpréter les énoncés ensemblistes les plus abstraits qui n'ont aucune chance de recevoir une quelconque application dans les sciences de la nature. La réponse est que leur vérité ou leur fausseté, démontrable ou indémontrable selon telle ou telle théorie, est indifférente au fonctionnement de notre appareil conceptuel et à notre compréhension de la nature telle qu'elle se reflète dans les énoncés catégoriques d'observations. Les mystères de ces énoncés sont ceux du développement et de l'usage de nos théories, ils sont propres au langage, non pas aux choses en elles-mêmes telles que la métaphysique se les représente.

\section{Conclusion}

Tous les développements du système de Quine sont loin d'avoir été présentés dans cette courte étude. Je me suis limité à exposer les arguments les plus importants. 
J'aborderai en guise de conclusion la question de la cohérence globale du système de Quine et je poserai aussi celle du progrès que Quine a apporté à la philosophie par sa réforme de l'empirisme.

On a reproché à ce système d'être victime de tensions internes ${ }^{64}$. On peut par exemple se demander comment concilier l'empirisme pour qui l'évidence sensorielle constitue à la fois la source de la connaissance et le moyen par lequel nous décidons finalement de ce qui existe, et le réalisme mathématique, qui admet la réalité des objets abstraits: en l'occurrence les classes ou les ensembles. Lorsque Quine admet que les mathématiques nous contraignent d'admettre l'existence d'une infinité de nombres réels que nous n'avons aucun moyen de désigner, il s'engage dans une ontologie qui dépasse les bornes de l'expérience sensible et il admet implicitement que le principe ultime de l'ontologie de son système est qu'être, c'est être un ensemble ou une chose ${ }^{65}$. On peut alors se demander comment concilier le réalisme mathématique avec l'idée empiriste selon laquelle «toute preuve qu'il peut y avoir pour la science est d'ordre sensoriel66», tant le rapport des objets abstraits non-spécifiables avec une quelconque preuve sensorielle apparaît pour le moins problématique. Un platonicien plus authentique comme Gödel a utilisé la métaphore des «yeux de l'esprit » pour exprimer cet accès de l'esprit au monde intelligible des objets mathématiques. Rien de comparable chez Quine, qui reconnaît à regret la nécessité de la quantification sur les ensembles. Mais cette réticence trahit-elle une véritable gêne théorique? C'est ce que je ne crois pas.

À la question de savoir comment rendre compte de l'existence de ces objets qui transcendent tout moyen d'expression dans le cadre de l'empirisme, Quine répond que celle-ci est impliquée par l'axiomatique qui est au fondement de la construction de l'ensemble des nombres réels, et par rien d'autre. La conséquence logique est aussi, avec la preuve sensorielle, une justification naturelle de la vérité des énoncés. On pourrait peut-être alors s'inquiéter de la pertinence de la cri- 
tique de la distinction de l'analytique et du synthétique, cependant on a déjà vu que Quine ne contestait pas cette distinction mais uniquement son caractère absolu: l'existence d'une infinité de nombres réels non spécifiables est une conséquence incontestable de la théorie des nombres; elle est donc analytique, mais c'est une conséquence analytique d'une théorie donnée et non une vérité analytique tout court.

Le problème soulevé par la critique anti-empiriste évoquée plus haut provient d'une confusion entre la portée de la thèse empiriste elle même, et celle de l'engagement ontologique d'une théorie. L'empirisme est une thèse épistémologique au sens restreint du terme, c'est-à-dire une thèse qui porte exclusivement sur la question de notre mode d'accès aux objets de la connaissance, celui-ci ne pouvant être que sensoriel. Si complexes soient les processus neuronaux par lesquels les mathématiques s'élaborent, il n'est pas douteux qu'ils existent et qu'ils sont la condition de la formulation des énoncés mathématiques. L'ontologie d'une théorie répond à la question de savoir qu'elles sont les valeurs qu'une théorie admet pour être vraie, et cette question est totalement indépendante de celle de notre mode d'accès à la connaissance de ces valeurs. Autrement dit, même si l'association de l'empirisme et du réalisme mathématique est rare dans l'histoire de la philosophie, elle ne peut pas être considérée comme incohérente, ni même comme instable ou inauthentique. D'autre part, l'existence transcendante d'une infinité de nombres ou d'ensembles peut très bien recevoir une signification empirique, comme celle par exemple de l'idée d'une approximation toujours plus fine de la mesure physique, ou plus précisément encore, celle de la continuité de la temporalité ou de l'espace.

L'existence de ces objets abstraits qu'il est impossible de spécifier n'oblige donc pas à conclure qu'il existe autre chose que le monde physique et le langage qui est lui aussi un phénomène physique. Quine admet la thèse du monisme anomal défini par Davidson, selon laquelle si seuls existent les corps et leurs états, tous les prédicats mentalistes qui groupent ces états ne sont pas traductibles en termes physiologiques ${ }^{67}$. Mais ce dua- 
lisme des prédicats mentalistes et des prédicats physicalistes n'implique ni l'abandon de l'empirisme, ni l'abandon de ce monisme de principe, ni l'accord avec la thèse platonicienne assumant l'existence d'un monde intelligible indépendant du monde sensible.

On a aussi reproché à Quine d'avoir donné une définition trop lâche ou trop vague de son holisme, et finalement d'échouer à nous faire comprendre comment la science se constitue. Quine aurait analysé la physique et les sciences de la nature en logicien, négligeant le fait que la constitution des sciences de la nature s'appuie sur des ressources bien plus riches que celle de la logique. Le reproche est d'une certaine façon admis par Quine puisqu'il reconnaît que, si la science répond toujours de quelque façon à la stimulation sensorielle à la fois à son début et plus tard, son mode de réponse après la rupture des amarres échappe au schématisme de son analyse ${ }^{68}$. Mais ce qui apparaît comme une concession n'est pas le constat d'un échec. C'est en philosophe et en logicien que Quine donne cette théorie de la connaissance qui schématise l'essentiel ${ }^{69}$; il revient aux autres spécialistes des sciences de la nature de poursuivre le travail de naturalisation de l'épistémologie et d'apporter leur contribution à la compréhension positive de la manière dont nous comprenons le monde qui nous entoure.

Inspirée par la science et guidée par la logique mathématique, l'œuvre philosophique de Quine apporte-t-elle un réel progrès philosophique? Sans doute faudrait-il s'interroger sur la légitimité de l'idée même de progrès philosophique, mais j'évite cette question préalable puisque Quine considère qu'il est vain de chercher à distinguer nettement science et philosophie. Quine a répondu positivement à cette question du progrès philosophique: celui-ci est celui de l'empirisme qui a fait disparaître certaines questions philosophiques en cinq étapes majeures, comme il l'explique dans «Five Milestones of Empricism ${ }^{70}$ ». La première est celle de l'abandon de la croyance en l'identité des idées qui était propre au conceptualisme de Locke. La seconde est le fait que la 
sémantique a déplacé son centre d'intérêt des termes vers les énoncés. La troisième est un autre déplacement de ce centre d'intérêt jusqu'aux systèmes d'énoncés. La quatrième est l'abandon du dualisme de l'analytique et du synthétique. La cinquième est le naturalisme, c'est-à-dire l'abandon de la recherche d'une philosophie première au profit de la science de la nature. La question de savoir si Quine a apporté un progrès en philosophie revient donc à savoir s'il faut approuver ou nier ces thèses fondamentales qui ont été ici brièvement exposées. La philosophie analytique contemporaine n'a cessé de débattre de ces questions. La perspective, du point de vue du système de Quine, a elle- même progressé et s'est raffinée. Personne n'a, à ma connaissance, scientifiquement démontré que Quine avait commis des erreurs décisives susceptibles de ruiner une de ces cinq thèses. On peut donc considérer qu'il y a progrès, à moins d'établir une frontière infranchissable entre science et philosophie.

\section{NOTES}

1. Gloria Origgi, Introduzione a Quine, Giuseppe Laterza \& Figli, RomaBari, 2000, p. 3.

2. Voir par exemple Pursuit ot Truth, Cambridge, Harvard University Press, 1990; trad. M. Clavelin, La poursuite de la vérité, Seuil, Paris, 1993. Voir aussi From Stimulus to Science, Cambridge, Harvard UP, 1995.

3. «Ma position méthodologique sur les fonctions délégantes et l'inscrutabilité de la référence doit aussi être comprise comme l'expression de mon naturalisme [...]» («Things and Their Place in Theories», Theories and Things, Cambridge, Harvard UP, 1981, p. 21).

4. Je dois pour cette section beaucoup à Roger Gibson, «Quine on the Naturalizing of Epistemology», dans On Quine, News Essays, Leonardi \& Santambrogio eds., Cambridge, Cambridge UP, 1995, p. 89-103.

5. Quine, La Poursuite de la Vérité, p. 43.

6. Quine, La Poursuite de la Vérité, p. 21. Les premières lignes de The Roots of Reference, La Salle, Open Court, 1974, et de From Stimulus to Science, cit., soulèvent la même question. 
7. Quine, «Reactions", dans On Quine, New Essays, Leonardi \& Santambrogio eds, cit., p. 349.

8. Sur le caractère scientifique de l'empirisme propre à l'épistémologie naturelle, voir Le Mot et la Chose, tr. fr. P. Gochet, Flammarion, Paris, 1977, rééd. Coll. «Champs» Paris, 1999, p. 29.

9. Quine, «Empirical Content», in Theories and Things, cit., p. 24.

10. Gibson, 1995, p. 90.

11. Quine, La Poursuite de la Vérité, p. 43 et p. 74. Quine a reconnu avoir user du terme «irritations de surface», «comme pour décourager une interprétation phénoméniste »; l'usage de l'expression «excitation des terminaisons nerveuses dans Word and Object, obéit au même esprit: «Personne ne pourrait supposer que j'imaginais que les gens pensent en général à l'excitation de leurs terminaisons nerveuses ni en parlent; peu de gens, statistiquement parlant, savent quelque chose au sujet de leur terminaisons nerveuses.» («On the Very Idea of a Third Dogma», in Theories and Things, cit., p. 40).

12. Sur ce rapprochement avec Russell, voir Le Mot et la Chose, p. 92-97.

13. Quine, Le Mot et la Chose, p. 65-66.

14. Quine, La Poursuite de la Vérité, § 2 à 4, p. 23-34.

15. Quine, 1990, trad. fr. 1993, p. 74.

16. Quine, Le Mot et la Chose, p. 66.

17. Quine, "Reactions» in Leonardi \& Santambrogio eds., 1995, p. 349. Voir aussi Quine, From Stimulus to Science, cit., chap. 2, p. 15-26.

18. «Avec Took et Bentham commença alors la sérieuse externalisation de l'empirisme: le déplacement de l'intérêt pour les idées, qui sont subjectives, vers le langage, qui est intersubjectif et social.» («Replies, to Chomsky », dans Words and Objection, Davidson \& Hintikka eds., Synthese Library, vol. 21, 1969, 1975, p. 306.)

19. Gibson, 1995, p. 91.

20. Quine, «L'épistémologie devenue naturelle », in Relativité de l'ontologie et autres essais, trad. Largeault, Aubier-Montaigne, Paris, 1977, p. 83-105.

21. Quine écrit: «Il y a relation de contenant à contenu, et réciproquement, bien qu'en des acceptions différentes, de l'épistémologie dans la science de la nature, et de la science de la nature dans l'épistémologie.» ( «'épistémologie devenue naturelle», p. 97)

22. Quine, «L'épistémologie devenue naturelle», p. 89.

23. Quine, From Stimulus to Science, cit., ch. IV, p. 43-50.

24. Quine, «Empirical Content», in Theories and Things, p. 28.

25. Quine, "On the Very Idea of a Third Dogma", in Theories and Things, cit., p. $38-42$. 
26. Il serait cependant faux de soutenir qu'aucun énoncé d'une théorie n'a de signification empirique qui lui soit propre: les énoncés d'observations sur lesquels s'établissent les énoncés théoriques déterminent graduellement la signification empirique propre d'un énoncé de la théorie. (cf. Quine, «Five Milestones of Empiricism », Theories and Things, cit., p. 71) Le partage du contenu empirique n'est pas la confusion des significations des énoncés.

27. «L'argument essentiel qui est dessous ma thèse de l'indétermination de la traduction, était qu'un énoncé sur le monde n'a pas toujours, ou bien n'a pas ordinairement, un fonds séparable de conséquences empiriques qui lui est propre.» (Quine, «L'épistémologie devenue naturelle », Relativité de l'ontologie et autres essais, cit., p 96.) Même si Quine fait ici référence à la théorie de l'indétermination de la traduction, il est préférable de corriger en précisant que c'est la théorie de la sous-détermination empirique des théories scientifiques dont il s'agit dans ce cas. Sur la distinction et les parallèles que l'on peut établir entre ces deux théories voir le $\$ 43$ de La Poursuite de la Vérité, p. 143-144, ainsi que «Indeterminacy of translation again », Journal of Philosophy, 1987, 84, p. 15-24.

28. Philosophie de la Logique, trad. Largeault, Aubier-Montaigne, Paris, 1975, ch. 1, p. 14.

29. Quine, «L'épistémologie devenue naturelle», Relativité de l'ontologie et autres esssais, cit., p. 90-97.

30. Quine, «L'épistémologie devenue naturelle», Relativité de l'ontologie et autres essais, cit., p. 96.

31. La Poursuite de la Vérité fait référence à la neurologie, à la psychologie, à la psycholinguistique, à la génétique évolutionniste, aux éclairages anecdotiques de l'histoire des sciences, et à la statistique mathématique appliquée (cf. p. 21 et 44).

32. Quine, «L'épistémologie devenue naturelle», Relativité de l'ontologie et autres essais, cit., p. 98. p. 91.

33. Quine, The Roots of Reference, Open Court, 1990, p. 2-3; Gibson1995,

34. Pour un examen de ces critiques, voir Gochet, Ascent to Truth, A Critical Examination of Quine's Philosophy, Philosophia Verlag, 1986, p. 29-34.

35. Quine, La Poursuite de la Vérité, p. 45-46.

36. Quine, «Relativité de l'Ontologie», Relativité de l'ontologie et autres essais, cit., p. 39.

37. Quine, La Poursuite de la Vérité, p. 66

38. Chomsky, «Quine's Empirical Assumptions », in Words and Objections, cit., p. 53-68. Voir la réponse de Quine, «Replies», ibid., p. 302-311. 
39. Quine, «Linguistics and Philosophy », The Ways of Paradox and other essays, Cambridge, Harvard Univ. Press, 1966, 1976, p. 56-58.

40. Quine, «Espèces naturelles», Relativité de l'ontologie et autres essais, cit., p. 140-141.

41. Rappelons la différence entre intention (intentionalité, intentionnel) c'est-à-dire la direction consciente d'une pensée vers un objet et intension qui fait référence à la signification d'une expression linguistique. Que le langage implique l'intentionalité et que celle-ci puisse exister indépendamment de luilà n'est pas ce que conteste Quine, mais l'existence d'intensions pré-linguistiques ainsi que l'utilité à en postuler dans une théorie de la communication.

42. Quine, «Relativité de l'ontologie », in Relativité de l'ontologie et autres essais, cit., p. 40.

43. Russell, An Inquiry into Meanging and Truth, Allen \& Unwin, 1950, Signification et Vérité, trad. Devaux, Flammarion, Paris, 1969, p. 294-295.

44. Quine, La Poursuite de la Vérité, p. 92.

45. Le Mot et la Chose, p. 83, note 1.

46. Quine, Philosophy of Logic, Prentice Hall, 1970, trad. fr. Largeault, Philosophie de la Logique, Aubier, Paris, 1975, p. 18-21.

47. Voir La Poursuite de la Vérité, p. 88.

48. Quine, La poursuite de la Vérité, p. 81.

49. Quine, La Poursuite de la Vérité, p. 65-66.

50. Quine, Ontological Relativity and Other Essays, Columbia University Press, New-York et Londres, 1969; trad. fr. Largeault, Relativité de l'Ontologie et autres essais, cit., p. 43.

51. Ibid., p. 48.

52. Quine, La poursuite de la vérité, § 13, p. 60.

53. Ibid., p. 81-82.

54. Gochet, 1986, p. 63.

55. Quine, La poursuite de la vérité, p. 137.

56. Quine, «On Carnap Views on Ontology», in The Ways of Paradox and Other Essays, cit., p. 203-211.

57. Quine, «Existence et quantification", in Relativité de l'ontologie et autres essais, cit., p. 124.

58. Quine, Le Mot et la Chose, cit., §31, p. 218.

59. Quine, «Reference and Modality», in From a Logical Point of View, Cambridge, Harvard Univ. Press, 1953, p. 139-159.

60. Quine, «Quantifiers and Propositional Attitudes», in The Ways of Paradox and Other Essays, cit., p. 185-196. 
61. Quine, Méthode de Logique, chap. 43, p. 253.

62. Quine, Quiddités, Article «Universaux», p. 250-254.

63. Quine, «New Foundations for Mathematical Logic», American Mathematical Monthly, 44, p. 70-80, repris dans From a Logical Point of View, cit., p. 80-101.

64. Sur ces critiques et une réponse à ces critiques, voir Gochet, 1986, p. 28-34.

65. Vuillemin, What are philosophical systems, Cambridge, Cambridge Univ. Press, 1986, p. 133.

66. Quine, «L'épistémologie devenue naturelle», p. 89. Déjà cité dans la section 1.

67. Quine, La poursuite de la vérité, p. 105-107, et From Stimulus to Science, p. $87-88$.

68. Quine, La poursuite de la vérité, p. 42.

69. Ibid., p. 22.

70. Quine, «Five Milestones of Empiricism », in Theories and Things, cit., p. $67-72$. 